\title{
Transoral Robotic Surgery and Oropharyngeal Tumors: Key Points in the Imaging Presurgical Workup
}

\begin{abstract}
Antonello Vidiri MD ${ }^{1}$, Jacopo Zocchi MD' ${ }^{2}$, Francesca Piludu MD', Silvia Moretto MD², Gerardo Petruzzi MD², Julia Daffinà ${ }^{1}$, Marcello Greco $\mathrm{MD}^{1}$, Francesco Mazzola $\mathrm{MD}^{2}$, Barbara Pichi $\mathrm{MD}^{2}$, Valentina Manciocco $\mathrm{MD}^{2}$, Armando De Virgilio $\mathrm{MD}^{3,4}$ and Raul Pellini MD ${ }^{2}$
\end{abstract}

${ }^{1}$ Radiology and Diagnostic Imaging Department, IRCCS Regina Elena National Cancer Institute, Via Elio Chianesi 53, 00144 Rome, Italy

${ }^{2}$ Department of Otolaryngology \& Head and Neck Surgery, IRCCS Regina Elena National Cancer Institute, Via Elio Chianesi 53, 00144 Rome, Italy

${ }^{3}$ Humanitas University, Via Rita Levi Montalcini, 4, 20090 Pieve Emanuele (MI), Italy

${ }^{4}$ Department of Otorhinolaryngology Head and Neck Surgery, IRCCS Humanitas

Clinical and Research Center, Via Alessandro Manzoni, 56, 20089 Rozzano (MI), Italy

*Corresponding Author: Antonello Vidiri MD, IRCCS Regina Elena National

Cancer Institute, Department of Radiology, via Chianesi 53, 00144 Rome, Italy

E-mail: antonello.vidiri@ifo.gov.it
Received: April 26, 2021

Published: May 19, 2021

(C) All rights are reserved by Antonello Vidiri., et al.

\begin{abstract}
Background: A major comprehension of the morphologic and radiologic anatomy of this region pushed the evolution and diffusion of mini-invasive techniques for the treatment of oropharyngeal benign and malignant lesions in the last decade.

Methods: We propose a radiological checklist for a systematic evaluation of the oropharyngeal region, with a particular reference to oropharyngeal squamous cell carcinoma (OPSCC) and its patterns of diffusion.

Results: Palatine tonsil, base of the tongue, soft palate and posterior oropharyngeal wall anatomy are thoroughly described with particular attention to the structures that need to be evaluated before a transoral surgical approach is considered.

Conclusion: Detailed preoperative evaluation of oropharynx anatomy is essential prior to any transoral approach for neoplastic disease. Routine use of a checklist strengthens multidisciplinary collaboration, supports a mutual professional growth and facilitates the analysis of treatment functional and oncological outcomes.
\end{abstract}

Keywords: TORS; Oropharynx; MR

\section{Introduction}

Last decades have seen the advent of new minimal invasive surgical approach. In the field of head and neck oncology, transoral robotic surgery (TORS) has become a valuable alternative to traditional external surgical approaches. The oropharynx represents the anatomical site most commonly addressed with this technique [1], although its use is now consolidated for other subsites such as the supraglottic larynx and the parapharyngeal space $[2,3]$.

TORS is particularly indicated in T1/T2 N0/N1 oropharyngeal squamous cell carcinoma (OPSCC) [4,5]; selected locally advanced and recurrent/persistent OPSCC; in the diagnostic work-up of unknown primary tumors [6,7]; in the treatment of Obstructive Sleep Apnea and benign lesions [8]; in selected cases of retropharyngeal lymph nodes dissection for diagnostic and therapeutic purposes [9].

The choice of a transoral approach relies on a proper pre-surgical study. This consists of a clinical evaluation of both the lesion, during the physical examination with the support of endoscopy and NBI techniques [10], and the patient, in order to exclude anatomical factors limiting the transoral approach. Of greater impor- 
tance, radiological imaging provides more details about the position and anatomical variants of the main vascular trunk and the real extension of a neoplastic lesion, in particular considering the possible deep spaces involvement. An accurate preoperative radiological investigation is crucial to define the indication and the kind of resection to perform and predict anatomical variants and safely manage the main vascular structures [11]. In this setting, cooperation between surgeons and radiologists and exchange of mutual competences is of primary importance in order to achieve the best management possible.

The purpose of this work is to draw up a radiological check list of the oropharyngeal regions which must be evaluated and described by radiologists in order to guide the surgeon dealing with TORS.

\section{Proposal of checklist}

The anatomical limits of oropharynx are represented by the soft palate superiorly, the glosso-epiglottic vallecula inferiorly, a line passing through the circumvallatae papillae of the tongue and the anterior tonsillar pillars anteriorly, the tonsillar fossae and pharyngeal wall laterally and posteriorly respectively. The authors propose a methodical, step-by-step approach focused on oropharyngeal anatomy, from the surgical transoral perspective.

Even if a detailed description of imaging techniques is beyond the purpose of this document, some preliminary observations are essential to deal with images in a proper way.

Multidetector Computed Tomography (CT) and Magnetic Resonance Imaging (MRI) represent the two radiological techniques available to obtain morphological information of the oropharyngeal region and in the evaluation of submucosal extension of neoplasms [12,13].

MRI with gadolinium should be considered the gold standard for the evaluation of the OPSCC, both for early and advanced staged lesions, in relation to the high contrast resolution, for which MRI is also employed for locoregional post-treatment surveillance $[14,15]$. CT with contrast medium is selected for patients who have contraindications to MRI, who are not collaborating (tests limited by movement artifacts) or when MRI is not available; CT permits to obtain volumetric acquisition and 3D reconstruction. PET-CT can be integrated in case of advanced staged neoplasms or unknown primary [16]. The identification of a small primary lesion, however, can be limited by the presence of lymphoid tissue both in the palatine tonsil and in the base of tongue [17], regardless of the technique employed.
On MRI and CT, the line that permits to differentiate oral cavity and oropharynx is at the junction between hard and soft palate. Acquisition parameters and choice of correct plane orientation is very important in order to allow an accurate evaluation of the oropharynx region.

\section{Palatine tonsil}

The lateral oropharyngeal wall consists of the anterior and posterior tonsillar pillars, the palatoglossal and palatopharyngeal muscles respectively, delimiting the tonsillar fossa. In an inside-out view, behind the tonsillar fossa we encounter the pharyngobasilar fascia, the superior constrictor muscle and the buccopharyngeal fascia, which covers anteriorly the medial pterygoid muscle and posteriorly the parapharyngeal fat (Figure 1). From the tonsillar fossa, OPSCC can spread inferiorly through the amigdaloglossus sulcus into the base of the tongue, laterally beyond the superior constrictor muscle into the pre-styloid space, masticator space and mandible, postero-laterally into the post-styloid space, posteriorly into the retropharyngeal space and superiorly into the rinopharynx and into the skull base.

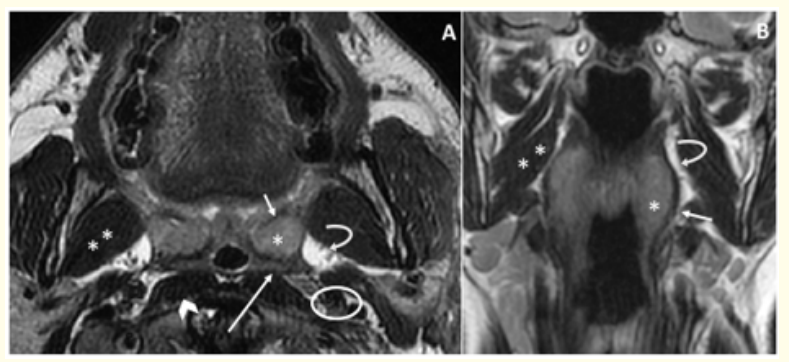

Figure 1: T2-weighted MRI axial (A) and coronal (B) planes. Normal anatomy: palatine tonsil (asterisk); palatoglossus muscle (short arrow) superior constrictor muscle (long arrow); parapharyngeal space (curved arrow); medial pterygoid muscle (double asterisk); carotid space (circle); prevertebral muscle (arrowhead).

Even if TORS is mainly indicated for lesions limited to the tonsillar fossa, the involvement of the superior constrictor muscle and of the pre-styloid or post-styloid parapharyngeal space doesn't represent an absolute contraindication [11,18], especially in case of non-malignant tumors (Table 1).

CT and MRI better evidence the palatine tonsil on axial and coronal planes; in particular they provide information on the dimension, the symmetry and can even detect the presence of small lesions. An accurate comparison between the two tonsils is im- 
Transoral Robotic Surgery and Oropharyngeal Tumors: Key Points in the Imaging Presurgical Workup

\begin{tabular}{|c|c|c|c|}
\hline Parameters & Check & CT/MRI evaluation & TORS \\
\hline \multicolumn{4}{|l|}{ Palatine tonsil } \\
\hline Superior Constrictor Muscle & No___ Yes__ & Axial/coronal & Yes \\
\hline Pre Styloid space & No___ Yes__ & Axial/coronal & Yes \\
\hline Post Styloid space & No__ Yes__ & Axial & No \\
\hline Base of the Tongue & No___ Yes__ & Axial/sagittal & Yes \\
\hline Retromolar Trigone & No__ Yes__ & Axial/coronal & No \\
\hline Pre Vertebral Fascia/Muscles & No___ Yes__ & Axial/sagittal & No \\
\hline Rinopharynx & No___ Yes__ & Axial/coronal & No \\
\hline Skull Base & No__ Yes__ & Axial/coronal & No \\
\hline Soft Palate & No___ Yes__ & Axial/coronal & Yes \\
\hline Masticator Space & No___ Yes__ & Axial/coronal & Yes/No \\
\hline
\end{tabular}

Table 1: Imaging checklist before transoral surgery for palatin tonsil tumor and relative contraindications.

CT: Computed Tomography; MRI: Magnetic Resonance Imaging; TORS: Transoral Robotic Surgery; ICA: Internal Carotid Artery.

portant even for the not negligible chance (10\%) of bilateral cancer [6]. MRI is superior to CT in the evaluation of small lesions, in particular when contrast medium is employed, preferably adopting dynamic sequences: in this case, the neoplastic lesion appears as an area of enhancement inside the tonsillar parenchyma. To define the limits of the tumor, it is preferable to use MRI with T2 weighted sequences for the muscles evaluation and contrast medium sequences for the submucosal spread. Radiologists have to rule out tonsillar superior pole, amigdaloglossus sulcus and posterior pharyngeal palatine arch involvement, as in these cases the surgical resection must move superiorly, medially and posteriorly respectively.

\section{Superior constrictor muscle}

MRI is superior to CT in the evaluation of the superior constrictor muscle that is located just beyond and laterally to the capsule of the palatine tonsil, covered by the pharyngobasilar fascia medially and the buccopharyngeal fascia laterally; this muscle appears as a linear low signal intensity on T2 sequences, around the palatine tonsil in axial and coronal planes; anteriorly the palatoglossus muscle can be observed. Evaluation of the superior constrictor muscle should be carefully reported, as an enlarged dissection is required in case of infiltration [19]. MRI shows the stretching or the infiltration of the superior constrictor muscle using the T2 sequences on axial and coronal planes.

\section{Pre-Styloid space}

Numerous structures that should be preserved are present in this area. The styloglossus and stylopharyngeal muscles and the stylohyoid ligament have to be recognised. The glossopharyngeal nerve passes through the styloglossus and stylopharyngeus muscles [20]. MRI and CT better show the parapharyngeal space on axial and coronal planes; on MRI, this space appears as an area of high signal intensity on T1 and T2 sequences, while on CT it appears as an hypodense area, due to the presence of fat tissue. The loss of hyperintensity on MRI and hypodensity on CT is correlated to the invasion of this space. The involvement of pre-styloid parapharyngeal space represents a relative contraindication, as a clear margins resection could be hard to achieve with a transoral approach [2] (Figure 2).

\section{Post-styloid space}

Distance from the posterior wall of the palatine fossa to the vas-

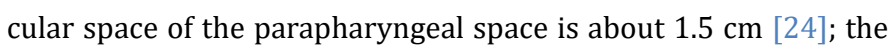
most important point to assess is the relationship between the lesion and the internal carotid artery (ICA), that is better evaluated in an axial plane; the radiological criteria to define the tumor-ICA relationships, in patients who did not undergo previous radiotherapy, is the degree of the circumferential involvement: an involvement superior to $270^{\circ}$ of the circumference of the carotid artery at MRI was considered as an evidence of the vessel infiltration [21] (Figure 3). 


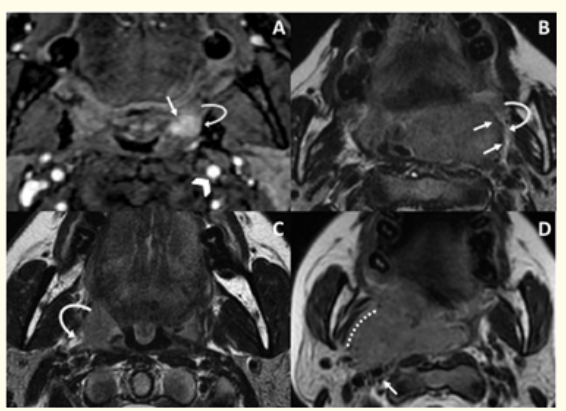

Figure 2: A: T1-weighted MRI with gadolinium, axial plane. SCC of the left palatine tonsil (short arrow), Adoptying our checklist we can observe that parapharyngeal space (curved arrow) and the carotid space (arrowhead) are not involved from by the lesion making this case suitable for TORS. B: T2-weighted MRI, axial plane, shows a large left tonsillar SCC with displacement of the superior constrictor muscle that is normal (arrows); parapharyngeal space (curved arrow) is free; TORS is possible. C: T2-weighted MRI, axial plane. A right tonsillar SCC: it is possible to appreciate the infiltration of superior constrictor muscle and parapharyngeal space (curved arrow). This represents a relative contraindication to TORS. D: T2-weighted MRI, axial plane. A large right tonsillar SCC with invasion of the parapharyngeal space and displacement of the medial pterygoid muscle (dotted line); the lesion shows a close relation to carotid artery (arrow); in this case the TORS is contraindicated.

SCC: squamous Cell Carcinoma; TORS: Transoral Robotic Surgery.

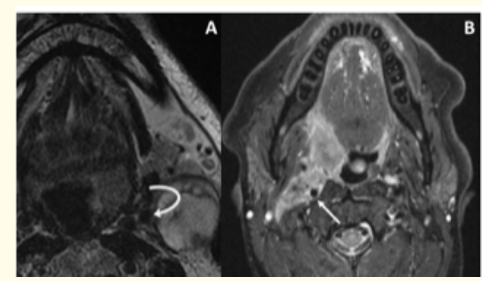

Figure 3: A: T2 weighted MRI, axial plane. A left tonsillar SCC. Note the retropharyngeal course of carotid artery (curved arrow). B: T1weighted MRI with gadolinium, axial plane, showing a SCC of right tonsil with an ipsilateral involvement of the post-styloid space and neoplastic encasement of internal carotid artery (arrow). TORS is contraindicated.

SCC: Squamous Cell Carcinoma; TORS: Transoral Robotic Surgery.
Independently from the degree of invasion, if OPSCC involves even minimally the parapharyngeal post-styloid space, adjacent buccopharyngeal fascia with part of the parapharyngeal fat should be resected. Most of the time this results in the ICA exposure which sometimes requires the reconstruction of the defect with a flap. Another aspect to evaluate is the course of the ICA, in particular the presence of an abnormal course of the artery which becomes retropharyngeal at the level of the oropharynx [22] (Figure 3). This increases the risk of damage during TORS, even if recent experiences demonstrated that this does not constitute an absolute contraindication for this approach [23]. Nevertheless, the presence of a tumor adjacent to the bulb or to the internal carotid artery, up to a complete encasement, should discourage TORS application [24].

\section{Base of the tongue}

The involvement of the base of the tongue is better outlined on axial and sagittal planes on $\mathrm{T} 2$ and $\mathrm{T} 1$ after contrast medium infusion at MRI or with CT after contrast medium with reconstructed plane on sagittal orientation (Figure 4).

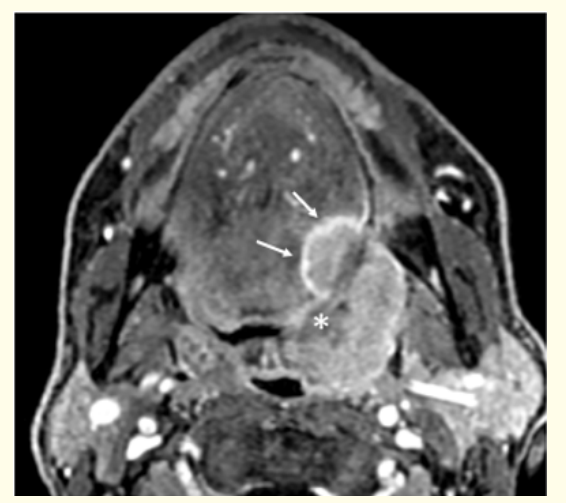

Figure 4: T1-weighted MRI with gadolinium, axial plane. A left tonsillar SCC with infiltration of the amigdaloglossus sulci (asterisk) and the left base of the tongue (arrows). The enhancing mass is indissociable from the left internal pterygoid muscle, completely invades the parapharyngeal space while it doesn't infiltrate the posterior oropharyngeal wall.

\section{Retromolar trigone}

Both the CT and MRI show well the invasion of the retromolar trigone, that is the mucosal region posterior to the last molar. The 
tumor infiltration of this region appears as the loss of hypointensity on CT and hyperintensity signal on T1 and T2 on MRI. In this case the lateral oropharyngectomy is extended anteriorly and can be conducted deep to the mandible periosteum including part of the medial pterygoid muscle. When this area is involved, in most of the cases a mandible involvement is present so that it must routinely be excluded. In the evaluation of this point, MRI is preferable because it is less affected by dental amalgam artifacts , which sometimes do not allow to define the exact extension of the tumor on the CT [25]. Mandible infiltration represents a contraindication to TORS.

Pre-vertebral space, fascia and muscles

MRI is superior to the CT to study the involvement of the prevertebral space, showing the loss of signal intensity of the fat on $\mathrm{T} 1$ and $\mathrm{T} 2$ sequences when infiltrated: this can be outlined both in axial and in sagittal planes. The preservation of the fat plane anteriorly to pre-vertebral muscles means that fascia is spared, even if it is challenging to demonstrate an infiltration at imaging exams [26]. In such a case, any surgical approach, including TORS, is not feasible. Retropharyngeal region contains the retropharyngeal lymph nodes, fibroadipose tissue, vascular and lymphatic vessels. Radiographic evaluation of the retropharyngeal nodes can be challenging in comparison to the lateral cervical nodes. Minimal axial diameter, superior to $6 \mathrm{~mm}$, focal nodal necrosis, ill-defined margins, 2 or multiple nodes are the criteria used by radiologist to define a node in this area [27]. MRI better shows small nodes into the retropharyngeal space, which appear as a round hypointense area in comparison to the hyperintense signal of the fat of the retropharyngeal space and show hyperintensity signals on diffusion MRI sequences. The nodes may be continuous with the primary tumor: this can represent a problem in particular in the CT evaluation where the nodes can be obscured by dental artifacts. For this reason, MRI is preferable and in doubt cases, PET-CT is advisable.

Nasopharynx

CT and MRI better evidence the involvement of this region in axial and coronal planes, whose infiltration represents a contraindication to TORS approach.

\section{Skull base}

CT shows the involvement of the skull base with the appearance of lytic lesions or an enlargement of the foramina in relation to the perineural diffusion. On MRI, the involvement of the skull base is evident in T1 and T2 sequences with a loss of hyperintense signal intensity of the medullary bone, replaced by low signal intensity on T1 and hyperintense signal on T2. MRI with 3D T1 sequences after contrast medium is the technique of choice in the evaluation of perineural diffusion, showing an enlargement and enhancement of the cranial nerve.

Soft palate

CT and MRI show the involvement of this region on axial and sagittal planes. In this case, the resection has to include the soft palate and its muscles (tensor veli palatini, levator veli palatini) up to the midline, even including the uvula.

\section{Masticator space}

The masticator space contains the mastication muscles (medial and lateral pterygoids, masseter, and temporalis) posterior mandible, and mandibular nerve. Infiltration of the muscles of the masticator space is better outlined on MRI on the T2 sequences on axial and coronal planes and on T1 sequences after contrast medium infusion. CT with contrast medium infusion can even demonstrate the infiltration of these muscles. Extensive tumor involvement of the masticator space, i.e. pterygoid or temporalis muscles, represent a contraindication for TORS approach as a radical removal cannot be achieved.

\section{Base of the tongue}

Base of tongue goes behind a line passing through the circumvallatae papillae, laterally is connected to the palatine tonsil through the amigdalo-glossus sulcus and inferiorly it continues with the glosso-epiglottic vallecula [28]. The tongue base consists of intrinsic and extrinsic muscles. Inferiorly, the lingual arteries run in the sublingual space. Lingual tonsillar tissue lies on the mucosal surface of the base of tongue (Figure 5).

\section{Extrinsic muscles of the tongue}

It is widely recognized that MRI is superior to CT in the demonstration of muscles involvement, in relation to the high contrast

Citation: Antonello Vidiri., et al. "Transoral Robotic Surgery and Oropharyngeal Tumors: Key Points in the Imaging Presurgical Workup". Acta Scientific Medical Sciences 5.6 (2021): 92-101. 


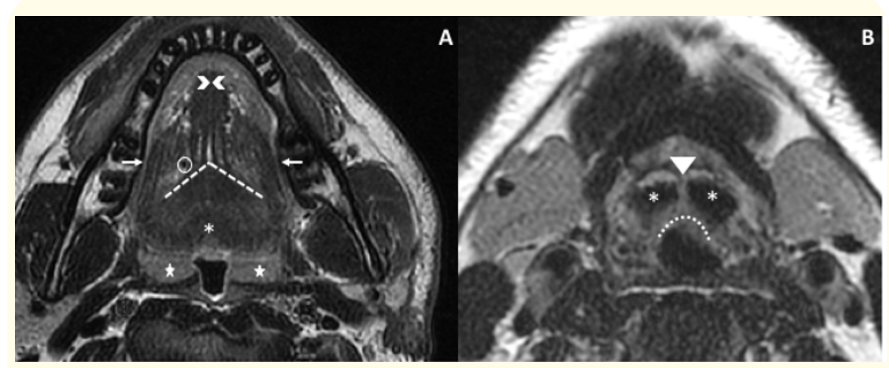

Figure 5: T2-weighted MRI, axial plane (A-B). Normal anatomy (A): base of the tongue (asterisk); V-shaped line of circumvallate papilla that ideally separates the base from the body of the tongue (dotted line), hyoglossus muscles (arrows); genioglossus muscles (arrowheads); lingual artery (circle); palatine tonsil (stars). Anatomy (B): glossoepiglottic vallecula (asterisks); glossoepiglottic fold (arrow head); epiglottis (dotted line).

resolution, on axial and coronal planes, in particular on the T2 sequences. MRI can demonstrate the infiltration of the styloglossus and hyoglossus muscles, which are thin structures, and the involvement of thicker ones such as the genioglossus (Figure 6 and 7). Infiltration of the hyoglossus muscle or extension beyond its boundary into the neck represents an absolute contraindication for TORS [29]; furthermore it is of crucial importance the evaluation of the distance of the tumor from the midline since the removal of more than half of the tongue is related to poor functional outcomes (Table 2).

\section{Lingual artery}

MRI and CT after contrast medium, in particular in the arterial phase, and US can show the course of the lingual artery in the presurgical planning [30]. Preoperative evaluation of the course of the lingual artery it is of crucial importance because its correct identification and ligation can prevent unexpected intraoperative bleedings and possibly prevent fatal post-operative hemorrhages. The involvement of the lingual artery can be hypothesized on the location of the vessel into the oropharynx and oral cavity that is lateral to the genioglossus muscles. While a monolateral involvement can be managed even with TORS, a bilateral encasement leads to devascularization of the tongue, thus representing a contraindication.

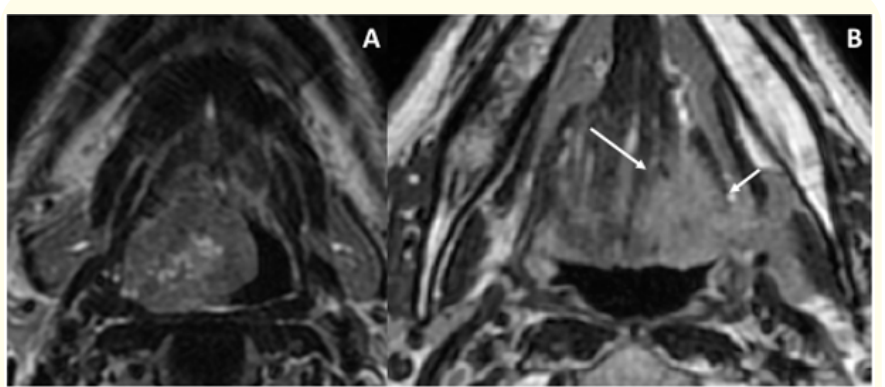

Figure 6: A: T2-weighted MRI, axial plane. SCC of the base of the tongue, determining an obliteration of the right epiglottic vallecula. It is possible to appreciate that anteriorly the extrinsic lingual musculature is not infiltrated and inferiorly the pre-epiglottic space is free while posteriorly the tumor rests on the superior constrictor muscle without infiltrating it. This case could be approached by TORS. B: T2-weighted MRI, axial plane. SCC localized into the left base of the tongue that infiltrates the extrinsic muscles (styloglossus and genioglossus, arrows). In this case TORS should be avoided.

SCC: squamous Cell Carcinoma; TORS: Transoral Surgery.

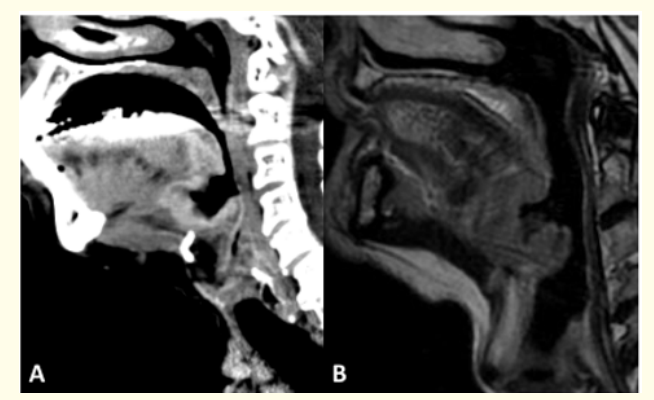

Figure 7: Contrast enhancement CT (A) and T2-weighted MRI (B), sagittal plane, in a patient with a SCC of the base of the tongue, infiltrating the extrinsic muscles. MRI shows a better contrast resolution compared to the CT (A).

\section{Mandible}

Involvement of the periosteum of the mandible is not amenable to transoral resection as there are currently no available transoral bone cutting instruments. CT and MRI are complementary for preoperative evaluation of mandibular bone invasion [31]. CT is superior to MRI to demonstrate the cortical bone erosion, in relation 
to the high spatial resolution. On the other side MRI is preferable for the evaluation of the medullary bone invasion in particular in edentulous patients, where the invasion occurs through the dental alveolus. MRI is superior to demonstrate the involvement of the mandibular canal and the perineural diffusion along the inferior alveolar nerve into V3, up to the oval foramen and the Gasser ganglion.

\section{Epiglottis}

MRI and CT better show the epiglottis infiltration on axial planes and sagittal plane.

\section{Pre-epiglottic space}

Extensive involvement of the pre-epiglottic fat tissue is also a relative contraindication due to the difficulty to achieve a clear margins resection and the intrinsic poor functional outcomes. The pre-epiglottic space is a fat-containing previsceral space and appears as an hypodense area on CT and as a hyperintense area on MRI on T1 and T2 sequences [32]. The involvement of this space is better outlined in sagittal reformatted plane on CT and in sagittal and axial planes on MRI. Infiltration is characterized by the loss of hypodensity on CT, replaced by enhanced tumor tissue; on MRI the high signal intensity of the fat on $\mathrm{T} 1$ and $\mathrm{T} 2$ sequences is replaced by the low signal intensity of the tumor on T1 and moderately hyperintense signal on $\mathrm{T} 2$.

\section{Hyoid bone}

Involvement of hyoid bone contraindicates TORS as a more demolitive surgery is needed in this case. CT and MRI show the involvement of hyoid bone on axial and sagittal planes; CT is superior to MRI in the evaluation of small lytic area into the hyoid bone.

\section{Soft palate}

CT and MRI better demonstrate this region on axial and sagittal planes, evidencing the possible hard palate infiltration anteriorly, when we prefer to use CT for the evaluation of the small lytic area in relation to the high spatial resolution in comparison to MRI. For the evaluation of perineural diffusion, in particular for minor gland tumors such as adenoid cystic carcinoma, the imaging of choice is MRI, that shows the perineural spread along palatine nerve into the pterygopalatine fossa (Table 3).

\begin{tabular}{|c|c|c|c|}
\hline Parameters & Check & CT/MRI evaluation & TORS \\
\hline \multicolumn{4}{|l|}{ Posterior wall } \\
\hline Retropharyngeal Space & No___ Yes__ & Axial/sagittal & Yes/No \\
\hline Pharyngobasilar Fascia & No___ Yes__ & Axial/sagittal & No \\
\hline Retropharyngeal nodes & No___ Yes__ & Axial/sagittal & Yes/No \\
\hline Retropharyngeal ICA & No___Yes__ & Axial/sagittal & Yes/No \\
\hline
\end{tabular}

Table 3: Imaging checklist before transoral surgery for oropharyngeal posterior wall tumor and relative contraindications. CT: Computed Tomography; MRI: Magnetic Resonance Imaging; TORS: Transoral Robotic Surgery; ICA: Internal Carotid Artery.

\section{Posterior wall}

In this region, it is very important to define the possible infiltration of the superior constrictor muscle, the retropharyngeal space and pharyngobasilar fascia (Table 4). In the evaluation of the involvement of these sites, MRI is superior to CT as previously reported (Figures 8).

\section{Exposure}

In the current literature, few anatomical characteristics that can predict an adequate exposure for TOS approach have been identi- fied. In a study by Luginbuhl et al., few factors resulted to be predictive for a good exposure: distance from posterior pharyngeal wall to hyoid $(<30 \mathrm{~mm})$, the angle between the epiglottis and vertical plane of the larynx $\left(\geq 130^{\circ}\right)$ and distance from the posterior pharyngeal wall to the soft palate $(\leq 8.1 \mathrm{~mm})$ [33]. It's the author's opinion that a thorough clinical physical examination can better provide predictability for sufficient robotic exposure: obesity, micrognathia, microstomia, trismus, teeth, tongue or craniofacial abnormalities are few of the factors to consider [33-35]. Further studies are needed to better predict the exposure of the oropharynx from a radiological point of view. 


\begin{tabular}{|c|c|c|c|}
\hline Parameters & Check & CT/MRI evaluation & TORS \\
\hline \multicolumn{4}{|l|}{ Soft palate } \\
\hline Hard Palate & No___ Yes___ & Axial/coronal/sagittal & No \\
\hline Nasal fossa & No___Yes__ & Axial/sagittal & No \\
\hline Rinopharynx & No___ Yes__ & Axial/coronal & No \\
\hline Pterygopalatine fossa & No___ Yes___ & Axial/coronal & No \\
\hline
\end{tabular}

Table 4: Imaging checklist before transoral surgery for soft palate tumor and relative contraindications. CT: Computed Tomography; MRI: Magnetic Resonance Imaging; TORS: Transoral Robotic Surgery.

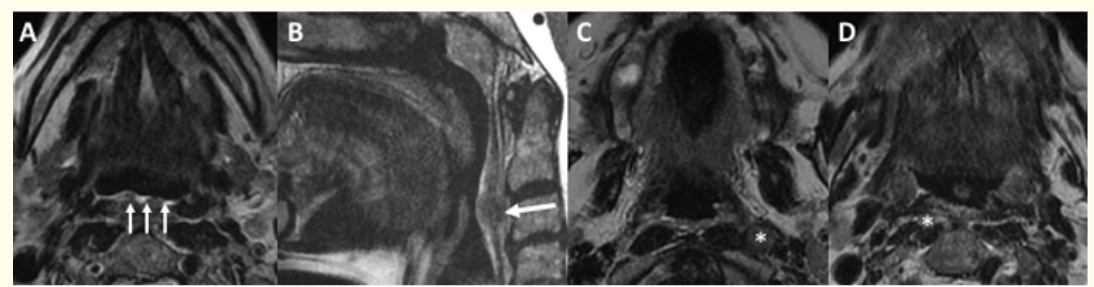

Figure 8: T2-weighted axial (A, C, D) and sagittal (B) MRI. A posterior wall SCC (A, arrows) with infiltration of the superior pharyngeal constrictor muscle and preveretebral fascia (B, arrow). Cases of a left retropharyngeal pathological node (C, dotted line) and a right node raising the ipsilateral superior constrictor muscle (D, dotted line). In such a case TORS is contraindicated.

\section{Discussion}

During the last decades, we assisted to the introduction and evolution of TORS in the field of head and neck oncology. Together with the larynx, the oropharynx represents the most suitable site to exploit the potentials of this techniques [1]. Several series have validated the feasibility of TORS, both for early and advanced OPC, reporting excellent oncologic outcomes [4]. The recent growth of the incidence of OPC, especially related to HPV, was accompanied by an increasing use of TORS, without lowering the standards of care [36].

The expansion to TORS indication requires as a prerequisite an optimal pre-surgical study, a clear understanding of the anatomy of the region and a consequent adaptation of the extent of the surgical resection. As usual in the field of the oncologic surgery, the main problem to face is the possibility to gain the radicality of the resection. This paper is intended as an aid for the radiologist but especially for the surgeon who considers TORS for the treatment of OPSCC: the first goal of a thorough radiological study is to determine local extension, in particular related to deep space involvement. A well-studied imaging allows to define the most suited indication, and when TORS is considered, to establish preoperatively the extent of resection, excluding those lesions not amenable with a pure transoral approach. At the same time, radiologist has to exclude the presence of pathological nodes at the level of the retropharyngeal [9] and cervical chain [37], that could change treatment indication.

In this sense, the checklist has the task of answering the surgeon's questions to the potential doubts that could be hidden behind transoral surgical approach.

The pre-operative collaboration between the radiologist and the surgeon should have the role of assuring a safer and planned surgery in order to minimize failures and reinterventions. At the same time, post-surgical feedback, sharing intraoperative and histopathological findings, favours a mutual professional growth.

Moreover, a routine use of such a checklist, with a clear description of the oropharyngeal subsites involved by the disease, will help to determine which patients are the best candidate for TORS through the evaluation of long term oncological and functional outcomes.

Citation: Antonello Vidiri., et al. "Transoral Robotic Surgery and Oropharyngeal Tumors: Key Points in the Imaging Presurgical Workup". Acta Scientific Medical Sciences 5.6 (2021): 92-101. 


\section{Conclusion}

Oropharyngeal cancer treatment represents one of the most debated topic in the current head and neck oncology literature. In this field, TORS has gained popularity in the last years providing a less invasive approach at comparable oncological outcomes. To maintain, or even raise the standard of care reachable with this technique, patient selection is a crucial prerequisite. A radiological checklist can represent a practical tool that can aid from the patient multidisciplinary evaluation to the surgical plan. Moreover, accurate definition of OPSCC extension, going beyond the TNM staging, will improve the analysis of long term outcomes.

\section{Bibliography}

1. Scholfield DW., et al. "Transoral Robotic Surgery for Oropharyngeal Squamous Cell Carcinoma: Improving Function While Maintaining Oncologic Outcome". Otolaryngology-Head and Neck Surgery 162.3 (2020): 267-268.

2. De Virgilio A., et al. "Trans-oral robotic surgery in the management of parapharyngeal space tumors: A systematic review". Oral Oncology 103 (2020): 104581.

3. Lechien JR., et al. "Surgical, clinical and functional outcomes of transoral robotic surgery for supraglottic laryngeal cancers: A systematic review". Oral Oncology 109 (2020): 104848.

4. de Almeida JR., et al. "Oncologic Outcomes After Transoral Robotic Surgery: A Multi-institutional Study". JAMA Otolaryngology - Head and Neck Surgery 141.12 (2015): 1043-1051.

5. Dabas S., et al. "Oncological outcome following de-intensification of treatment for stage I and II HPV negative oropharyngeal cancers with transoral robotic surgery (TORS): A prospective trial". Oral Oncology 69 (2017): 80-83.

6. Di Maio P., et al. "Role of palatine tonsillectomy in the diagnostic workup of head and neck squamous cell carcinoma of unknown primary origin: A systematic review and meta-analysis". Head and Neck 41.4 (2019): 1112-1121.

7. Patel SA., et al. "Robotic surgery for primary head and neck squamous cell carcinoma of unknown site". JAMA Otolaryngology - Head and Neck Surgery 139.11 (2013): 1203-1211.
8. Petruzzi G., et al. "Transoral Robotic Surgery in the Management of a Tongue Base Schwannoma". Journal of Craniofacial Surgery (2020).

9. Petruzzi G., et al. "Transoral robotic retropharyngeal lymph node dissection in a recurrent head and neck carcinoma". Head and Neck 41.11 (2019): 4051-4053.

10. Tirelli G., et al. "Tailored resections in oral and oropharyngeal cancer using narrow band imaging". American Journal of Otolaryngology 39.2 (2018): 197-203.

11. De Virgilio A., et al. "Anatomical-based classification for transoral lateral oropharyngectomy". Oral Oncology 99 (2019): 104450.

12. Trotta BM., et al. "Oral cavity and oropharyngeal squamous cell cancer: key imaging findings for staging and treatment planning". Neurologic/Head and Neck Imaging 31.2 (2011): 339-354.

13. Corey AS and Hudgins PA. "Radiographic imaging of human papillomavirus related carcinomas of the oropharynx". Head and Neck Pathology 6 (2012): S25-40.

14. Lenz M., et al. "Oropharynx, oral cavity, floor of the mouth: CT and MRI". European Journal of Radiology 33.3 (2010): 203-215.

15. Zocchi J., et al. "The role of a post therapeutic surveillance program for sinonasal malignancies: Analysis of 417 patients". Head and Neck (2019).

16. Golusinski P., et al. "Evidence for the approach to the diagnostic evaluation of squamous cell carcinoma occult primary tumors of the head and neck". Oral Oncology 88 (2019): 145-152.

17. Wong WL., et al. "18F-fluorodeoxyglucose positron emission tomography/computed tomography in the assessment of occult primary head and neck cancers--an audit and review of published studies". Clinical Oncology (R Coll Radiol). 24.3 (2012): 190-195.

18. Gun R., et al. "Transoral Surgical Anatomy and Clinical Considerations of Lateral Oropharyngeal Wall, Parapharyngeal Space, and Tongue Base". Otolaryngology-Head and Neck Surgery (2016). 
19. Kwan BYM., et al. "Transoral robotic surgery for head and neck malignancies: Imaging features in presurgical workup". Head and Neck 41.11 (2019): 4018-4025.

20. Gupta A., et al. "Imaging evaluation of the parapharyngeal space". Otolaryngologic Clinics of North America 45.6 (2012): 1223-1232.

21. Yousem DM., et al. "Carotid artery invasion by head and neck masses: prediction with MR imaging”. Radiology 195.3 (1995): 715-720.

22. Regloix SB De and Maurin 0. "Retropharyngeal course of the internal carotid artery". (2017): 2017-2018.

23. Gorphe P., et al. "Revisiting vascular contraindications for transoral robotic surgery for oropharyngeal cancer". Laryngoscope Investigative Otolaryngology 3.2 (2018): 121-126.

24. Baskin RM., et al. "Transoral robotic surgery for oropharyngeal cancer: patient selection and special considerations". Cancer Management and Research 10 (2018): 839-846.

25. Crecco M., et al. "Retromolar trigone tumors: evaluation by magnetic resonance imaging and correlation with pathological data". European Journal of Radiology 32.3 (1999): 182-188.

26. Hsu WC., et al. "Accuracy of magnetic resonance imaging in predicting absence of fixation of head and neck cancer to the prevertebral space". Head and Neck 27.2 (2005): 95-100.

27. Doumas S., et al. "Current thinking on the management of abnormal retropharyngeal nodes in patients with oral, oropharyngeal, and nasopharyngeal squamous cell carcinoma: a structured review". British Journal of Oral and Maxillofacial Surgery 57.6 (2019): 515-528.

28. Pellini R., et al. "Laryngeal vs oropharyngeal cancer: T2 or not T2". Oral Oncology 109 (2020): 104678.

29. Dallan I., et al. "Anatomical landmarks for transoral robotic tongue base surgery: comparison between endoscopic, external and radiological perspectives". Surgical and Radiologic Anatomy 35.1 (2013): 3-10.
30. Liu C., et al. "Ultrasonic Measurement of Lingual Artery and Its Application for Midline Glossectomy". Annals of Otology, Rhinology and Laryngology (2020): 3489420913581.

31. Bouhir S., et al. "Radiological assessment of mandibular invasion in squamous cell carcinoma of the oral cavity and oropharynx". European Annals of Otorhinolaryngology, Head and Neck Diseases 136.5 (2019): 361-366.

32. Corey A. "Pitfalls in the staging of cancer of the oropharyngeal squamous cell carcinoma". Neuroimaging Clinics of North America 23.1 (2013): 47-66.

33. Luginbuhl A., et al. "Preoperative cephalometric analysis to predict transoral robotic surgery exposure". Journal of Robotic Surgery 8.4 (2014): 313-317.

34. Arora A., et al. "Determination of biometric measures to evaluate patient suitability for transoral robotic surgery". Head and Neck 37.9 (2015): 1254-1260.

35. Rich JT., et al. "Transoral laser microsurgery (TLM) +/- adjuvant therapy for advanced stage oropharyngeal cancer: outcomes and prognostic factors". Laryngoscope 119.9 (2009): 1709-1719.

36. Cohen MA., et al. "Transoral robotic surgery and human papillomavirus status: Oncologic results". Head and Neck 33.4 (2011): 573-580.

37. Iocca O., et al. "Lymph node yield and lymph node ratio in oral cavity and oropharyngeal carcinoma: Preliminary results from a prospective, multicenter, international cohort". Oral Oncology 107 (2020).

\section{Volume 5 Issue 6 June 2021}

(C) All rights are reserved by Antonello Vidiri., et al. 